\title{
A Bi-population PSO with a Shake-Mechanism for Solving Constrained Numerical Optimization
}

\author{
Leticia C. Cagnina
}

\author{
Susana C. Esquivel
}

\author{
Carlos A. Coello Coello
}

\begin{abstract}
This paper presents an enhanced Particle Swarm Optimizer approach, which is designed to solve numerical constrained optimization problems. The approach uses a single method to handle different types of constraints (linear, nonlinear, equality or inequality) and it incorporates a shakemechanism and a dual population in an attempt to overcome the problem of premature convergence to local optima. The proposed algorithm is validated using standard test functions taken from the specialized literature and is compared with respect to algorithms representative of the state-of-the-art in the area. Our preliminary results indicate that our proposed approach is a highly competitive alternative to solve constrained optimization problems.
\end{abstract}

\section{INTRODUCTION}

The solution of constrained optimization problems using metaheuristics (particularly, evolutionary algorithms) has attracted a lot of interest in the last few years, since such problems are very common in real-world applications. One of the many metaheuristics that has been adopted to solve such problems is particle swarm optimization (PSO) [13]. PSO has been found to be highly competitive for solving unconstrained optimization problems [14], [15], [9], [3], [4]. However, its use in constrained optimization problems is still relatively scarce (see for example [19], [18], [16]).

PSO was conceived as a simulation of individual and social behavior [12] such as the one observed in flocks of birds and fish. PSO explores the search space using a population of individuals, and the best performers (either within a group or with respect to the entire population) affect the performance of the others. Each individual is named particle and represents a possible solution within a multidimensional search space. The particles have their own position and velocity, which are constantly updated. They record their past behavior and use it to move towards promising regions of the search space.

In this paper, we present a PSO algorithm which is designed to solve constrained optimization problems. For that sake, our approach contains a constraint-handling technique as well as a mechanism to update the velocity and position of the particles [2], which is extended by adding to it a bi-population and a shake-mechanism as a way to avoid premature convergence.

Leticia C. Cagnina and Susana C. Esquivel are with LIDIC (Research Group). Universidad Nacional de San Luis - Ej. de Los Andes 950 - (D5700HHW) San Luis, ARGENTINA. (emails: \{lcagnina,esquivel\}@unsl.edu.ar). Carlos A. Coello Coello is with CINVESTAV-IPN (Evolutionary Computation Group), Departamento de Computacion, Av. IPN No. 2508, Col. San Pedro Zacatenco, Mexico D.F. 07300, MEXICO. (email: ccoello@cs.cinvestav.mx).
The remainder of this paper is organized as follows. Section II provides the statement of general constrained optimization problems. Section III briefly discusses the previous related work. In Section IV, we describe in detail our proposed approach. Section V describes the experimental setup and provides an analysis of the results obtained from our empirical study. The conclusions and some directions for future research are stated in Section VII.

\section{Statement of the Problem}

Without loss of generality, we can consider the general nonlinear optimization problem as a minimization problem, which can be formally stated as the problem of finding $\vec{x}$ which:

$$
\operatorname{minf}(\vec{x}) \text { with } \vec{x}=\left(x_{1}, x_{2}, \ldots, x_{D}\right) \in \mathcal{F} \subseteq \mathcal{S} \subseteq \mathcal{R}^{D}
$$

subject to:

$$
\begin{array}{rl}
g_{i}(\vec{x}) \leq 0 & i=1,2, \ldots, n . \\
h_{e}(\vec{x})=0 & e=1,2, \ldots, m .
\end{array}
$$

Each $x_{d} \in\left[l_{d}, u_{d}\right]$ with $d \in[1 . . D]$. The $l_{d}$ and $u_{d}$ are the lower and upper bounds imposed on the decision variables. The $g_{i}$ and $h_{e}$ functions are defined on $\mathcal{S}$ (search space), and correspond to the inequality and equality constraint functions, respectively. A constraint delimits the search space splitting it into a feasible and an infeasible region. $\mathcal{S}$ is a D-dimensional rectangle defined by the lower and upper bounds of each variable $x_{d}$. All $\vec{x}$ satisfying all inequality and equality constraint functions determine the feasible solution space $\mathcal{F}$.

\section{Previous Related Work}

As indicated before, despite its success in a variety of optimization problems, the proposals of constraint-handling mechanisms for PSO are relatively scarce. Next, we will review the most representative work done in this area.

Zang et al. [23] presented a PSO algorithm that implements a periodic mechanism for handling constraints. The approach makes periodic copies of the search space when the algorithm starts the run, thus avoiding the disorganization that might arise when the mutation operator is applied to those particles lying on the boundary between the feasible and infeasible regions. The authors validated their algorithm with eight test functions and compared their results with respect to whose provided by conventional constraint-handling methods.

Toscano Pulido and Coello Coello [19] added to a basic PSO a simple mechanism for tackling constraints based on 
how close are the particles from the feasible region. A turbulence operator was incorporated in order to improve the exploration of the search space. The thirteen well-known constrained test functions from [21] were used to show the performance of this PSO algorithm. The authors concluded that their results were highly competitive.

Liang and Suganthan [16] proposed a novel constrainthandling mechanism based on multi-swarms (subpopulations). Each swarm works on a constraint, but these are assigned in an adaptive manner, and are periodically changed. Additionally, a local search mechanism is combined with the algorithm in order to improve the overall quality of the search performed. The authors validated their approach using twenty four test function taken from [8] and expressed their results in terms of Best functions error values. The authors reported that their results were satisfactory for 21 test functions, but the approach did not properly work in the other three.

Muñoz-Zavala et al. [18] presented a standard PSO algorithm improved with two perturbation operators that are only applied to the best particle population (pbest) at two different stages. These operators are used to keep the algorithm's exploration ability. To handle the constraints, they used the summation of constraint violations and a dynamic tolerance factor for the equality constraints (i.e., a higher tolerance is allowed at the beginning of the search, and such value is decreased along the run). The algorithm also maintains a "tolerant file", that is used when the perturbation operators are applied and the tolerance factor is decremented, in order to determine a better leader for the swarm. The authors also used the twenty four test functions from [8] to validate their approach. The results were found to be competitive, but the approach did not properly work in one of the test functions.

The algorithm CPSO-shake, which is proposed in this paper, shares some features with some of the algorithms cited above: the constraint-handling mechanism, the variant tolerance factor and the subpopulation concept. However, as we will see later on, the last two concepts are used in a different manner.

\section{CPSO-SHAKE ALGORITHM}

In this section, we describe in detail our proposed approach, which we call the CPSO-shake algorithm.

\section{A. General Model}

As stated before, a PSO algorithm operates on a population of particles. Due to the type of problem to optimize (with $n$ decision variables), the particles are $n$-dimensional real number vectors. The best position found so far for the particles (for the gbest model) or in the neighborhood (lbest model) is recorded. The best value reached by each particle (pbest) is stored, too. As in the standard model, the particles evolve using two update formulas, one for position and another one for velocity.

\section{B. Particular Model}

As it was stated in some of our previous work [1], the gbest model tends to converge to a local optimum; however, this model works well in a variety of problems. Motivated by this, we proposed a formula to update the velocity, using a combination of both the gbest and the lbest models [2]. Such a formula is adopted here as well, and is shown in equation (4).

$$
\begin{gathered}
v_{i d}=w\left(v_{i d}+\gamma_{1}\left(p_{i d}-p a r_{i d}\right)+\right. \\
\left.\gamma_{2}\left(p_{l d}-p a r_{i d}\right)+\gamma_{3}\left(p_{g d}-p a r_{i d}\right)\right)
\end{gathered}
$$

where $v_{i d}$ is the velocity of particle $i$ at the dimension $d$; $w$ is the inertia factor [5] whose goal is to balance global exploration and local exploitation. $\gamma_{1}$ is the personal learning factor, and $\gamma_{2}$ and $\gamma_{3}$ are the social learning factors. These 3 values are multiplied by 3 different random numbers within the range [0..1], $p_{i d}$ is the best position reached by the particle $i ; p_{l d}$ is the best position reached by any particle in the neighborhood, $p_{g d}$ is the best position reached by any particle in the swarm. par $_{i d}$ is the value of the particle $i$ at the dimension $d$.

But in [2] we modified the equation for updating the particles. In that paper, during $10 \%$ of the iterations, we applied the normal formula (depicted in equation (5)) as suggested in [13].

$$
\operatorname{par}_{i d}=\operatorname{par}_{i d}+v_{i d}
$$

And in the remainder $90 \%$ of cases, we used equation (6) proposed by Kennedy [11].

$$
\operatorname{par}_{i}=N\left(\frac{p_{i}+p_{l}}{2},\left|p_{i}-p_{l}\right|\right)
$$

where $p_{i}$ is the position of the particle to be updated, $N$ is the Gaussian random generator, $p_{i}$ and $p_{l}$ are the best position reached by the particle $p a r_{i}$ and the best position reached by any particle in the neighborhood of $\operatorname{par}_{i}$, respectively. That probability was empirically found to be the best after performing a series of experiments with all the test functions evaluated.

We use a circle topology [10] to compute the $p_{l d}$ value, in which each particle is connected to $k$ neighbors. The neighbors are determined by the position of the particles in the storage structure. Figure 1 illustrates this concept.

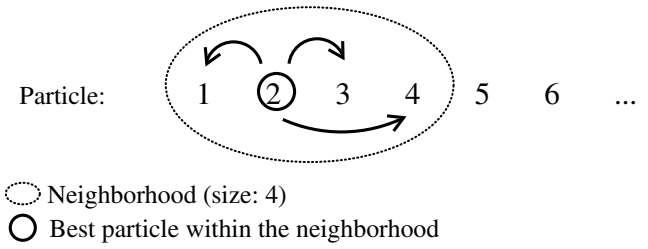

Fig. 1. Circle topology adopted in the population of our PSO approach 
To help avoiding convergence to a local optimum, we used a dynamic mutation operator [1] which is applied to each individual with a $p m$-probability. This value is calculated considering the total number of iterations in the algorithm (cycles) and the current cycle number as the following equation indicates:

$$
p m=m a x_{-} p m-\frac{m a x_{-} p m-m i n_{-} p m}{m a x_{\_} c y c l e} * c u r r e n t \_c y c l e
$$

where max_pm and min_pm are the maximum and minimum values that $p m$ can take, max_cycle is the total number of cycles that the algorithm will iterate, and current_cycle is the current cycle in the iterative process.

\section{Handling Constraints}

The constraint-handling scheme adopted in this paper is the simplest method possible to handle both equality and inequality constraints: we prefer a feasible particle over an infeasible one. When the two particles compared are infeasible, we choose the one which is closer to the feasible region. In order to do that, our algorithm stores the largest violation obtained for each constraint. When an individual is found infeasible, the amount of violation (this value is normalized with respect to the largest violation stored so far) is added. This strategy is used when the pbest, gbest and lbest particles are chosen. All equality constraints were transformed into inequalities, as is normally done in the evolutionary optimization literature, and we used a tolerance factor $(\epsilon)$ defined by the following formula:

$$
\left|h_{e}(\vec{x})\right|-\epsilon \leq 0
$$

\section{Dynamic Tolerance}

Our algorithm has a mechanism to adapt the value of $\epsilon$ during the run. When the search process starts, the value is initialized in 0.1 . As we increase the number of iterations, the value of $\epsilon$ is divided by 10 at three different moments (i.e., $\epsilon$ takes the values: $0.01,0.001$ and 0.0001 during a run). If we assume that $N$ is the total number of iterations to be performed, the value of $\epsilon$ will change according to the scheme graphically shown in Figure 2.

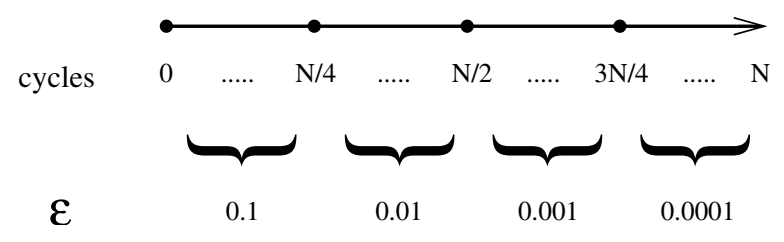

Fig. 2. Variation of $\epsilon$ during a run of our PSO approach

The main advantage of using a varying $\epsilon$ value is to favor the existence of feasible solutions at the beginning of the search process, so that the search space can be properly sampled (particularly in problems having equality constraints). As the number of iterations increases, $\epsilon$ is decreased, so that our approach starts converging towards solutions that satisfy the equality constraints with a higher numerical precision.

\section{E. Bi-population}

The CPSO-shake algorithm splits the entire population into two subpopulations each of which is independently evolved. The idea is to maintain more than one group of particles exploring the search space (at the same time). In that way the possibility of falling into local optima is reduced.

One may then wonder why to adopt only two subpopulations and not more. The reason is that it does not make any sense to adopt more than two subpopulations, considering the small number of particles that we use in our original population (only 10). In fact, we believe that our neighborhood topology would not work properly if we adopt less than 5 particles and therefore our choice of adopting only two subpopulations.

All the features stated before for the entire population (neighborhoods, lbest and gbest approaches, equations for updating the velocity and the positions) still apply, but in this case, they are applied not to a single population, but to each subpopulation. When the iterative process finishes, the best particle from both subpopulations is reported as the final output.

\section{F. Shake-Mechanism}

In our PSO-based proposal reported in [2], we had some stagnation problems when trying to obtain values close to the optima for some difficult test functions. In order to overcome this problem, the algorithm reported in this paper incorporates a shake-mechanism. This mechanism is applied when the percentage of infeasible individuals is higher than $10 \%$. This value was empirically derived. Note that it is not convenient to keep populations in which all the solutions are feasible, since infeasible solutions play an important role when trying to solve problems with active constraints [17].

In order to implement the shake-mechanism, some particles are moved to another place in the search space. Although this can be done by guiding a particle to a random direction, it is undesirable that the particles move away too much (we just want to shake them a little!). So, a particle with a good solution is selected as a reference: a randomly chosen pbest particle. Thus, equation (9) is used to move a particle $i$ :

$$
v_{i d}=w * v_{i d}+\gamma_{1} *\left(\text { psel }_{d}\right)
$$

where $v_{i d}$ is the $d$ th-position of the velocity vector, $w$ is the inertia factor, $\gamma_{1}$ is the personal learning factor multiplied by a random number within the range [0..1]. ssel $_{d}$ is the $d$ th-position of a (randomly chosen) pbest vector.

The shake-mechanism is applied with a $50 \%$ probability.

\section{G. CPSO-shake Pseudocode}

Figure 3 shows the pseudocode of our proposed CPSOshake algorithm. At the beginning of the search, we initialize the vectors of position and velocity of each particle in both subpopulations and we initialize the $\epsilon$ value, too (lines 2 to 6). 
After evaluating the particles and obtaining the best values: pbest, lbest and gbest (lines 7 and 8), the subpopulations begin to evolve. During the evolutionary process, new values of pbest, lbest and gbest are chosen and both, the velocity and the position of each particle are updated (lines 10 to 25). At line 26, a keeping mechanism is applied to control that all the dimensions in all particles are within the allowable bounds. We then calculate the percentage of infeasible individuals in the entire population (both subpopulations considered together) and apply the shake-mechanism if the required conditions are fulfilled (lines 27 to 30 ). The mutation probability is updated and the particles are mutated, if applicable (lines 31 and 32). After that, the particles are evaluated, new "best" values are recorded and the $\epsilon$ value is updated (lines 33 to $35)$. Finally, the best value reached by any subpopulation is taken and compared. The best of them is returned (lines 37 and 38).

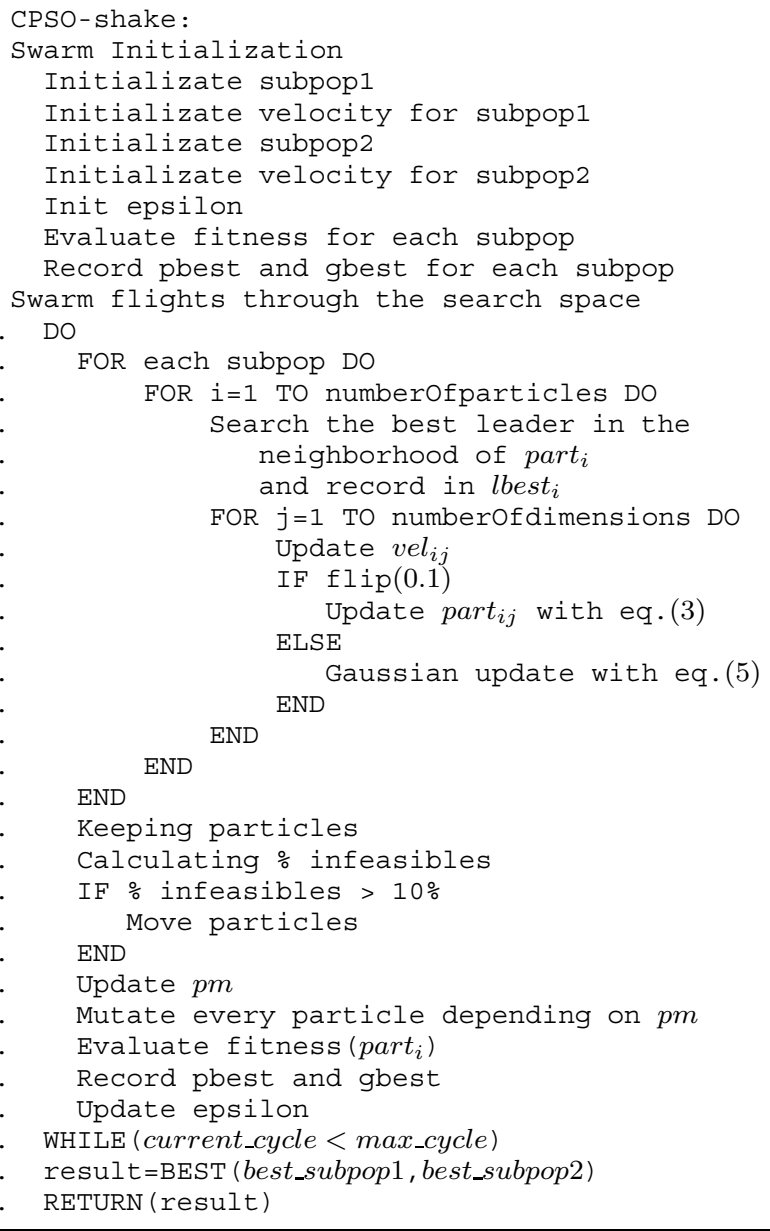

Fig. 3. Pseudo-code of CPSOshake

\section{EXPERIMENTAL STUDY}

For validating our proposed approach, we adopted 20 test problems taken from [8]. The detailed description of the test problems may be consulted in its original source [8].
TABLE I

COMPARISON OF THE best VALUES OBTAINED BY OUR CPSO-SHAKE AND THE APPROXIMATION EVOLUTION STRATEGY WHICH USES Stochastic RANKING (AESSR).

\begin{tabular}{|c||c||c|c|}
\hline Funct. & Benchmark & CPSO-shake & AESSR \\
\hline \hline $\mathrm{g} 1$ & -15.000 & -15.000 & -15.000 \\
\hline $\mathrm{g} 2$ & -0.803 & -0.803 & -0.739 \\
\hline $\mathrm{g} 3$ & -1.000 & -1.000 & -1.000 \\
\hline $\mathrm{g} 4$ & -30665.539 & -30665.538 & -30665.539 \\
\hline $\mathrm{g} 5$ & 5126.496 & 5126.498 & 5126.497 \\
\hline $\mathrm{g} 6$ & -6961.813 & -6961.825 & -6961.814 \\
\hline $\mathrm{g} 7$ & 24.306 & 24.309 & 24.306 \\
\hline $\mathrm{g} 8$ & -0.095 & -0.095 & -0.096 \\
\hline $\mathrm{g} 9$ & 680.630 & 680.630 & 680.630 \\
\hline $\mathrm{g} 10$ & 7049.248 & 7049.285 & 7049.408 \\
\hline $\mathrm{g} 11$ & 0.749 & 0.749 & 0.750 \\
\hline $\mathrm{g} 12$ & -1.000 & -1.000 & -1.000 \\
\hline $\mathrm{g} 13$ & 0.053 & 0.054 & 0.054 \\
\hline $\mathrm{g} 14$ & -47.764 & -47.635 & -47.765 \\
\hline $\mathrm{g} 15$ & 961.715 & 961.715 & 961.715 \\
\hline $\mathrm{g} 16$ & -1.905 & -1.905 & -1.905 \\
\hline g17 & 8853.539 & 8853.539 & 8853.540 \\
\hline $\mathrm{g} 18$ & -0.866 & -0.866 & -0.866 \\
\hline $\mathrm{g} 19$ & 32.655 & 34.018 & 32.665 \\
\hline g20 & 0.204 & 0.203 & - \\
\hline
\end{tabular}

All these test functions were transformed into minimization problems. All the equality constraints were transformed into inequalities. We performed 25 independent runs per problem, with a total of 350,000 evaluations of the objective function per run. Our proposed CPSO-shake used the following parameters: swarm size $=10$ particles, pm_min $=0.1$, pm_max $=0.4$, neighborhood size $=3$, inertia factor $w$ $=0.8$, personal learning factor and social learning factors for $\gamma_{1}, \gamma_{2}$ and $\gamma_{3}$ was set to 1.8. The parameter settings such as swarm size, mutation probability, neighborhood size and learning factors were empirically derived after numerous experiments.

Our results were compared with respect to those obtained by an Approximation Evolution Strategy which uses Stochastic Ranking (AESSR) recently proposed in [20] and with respect to the results produced by the Stochastic Ranking (SR) approach [21]. Both approaches are highly competitive and are representative of the state-of-the-art in the area. Note that in [20], the author validated his approach using 500,000 objective function evaluations per run, while our approach only performs 350,000 evaluations.

Table I shows the best values found by CPSO-shake and AESSR. The conclusion is the following: Despite the fact that the number of evaluations performed by our CPSOshake approach is $30 \%$ lower than the number of evaluations performed by AESSR, our CPSO-shake algorithm obtained results that are very satisfactory. For five functions (g2, g8, g10, g11 y g17) the values obtained by CPSO-shake are better than those obtained by AESSR. In 10 test functions, the results are the same and only for 4 test functions, AESSR obtained better values than CPSO-shake. All the solutions found by both algorithms are feasible, except for the value shown for g20 which is infeasible but is very close to the 
TABLE II

Best Values obtained with CPSO-ShaKe PERForming 350,000 OBJECTIVE FUNCTION EVALUATIONS.

\begin{tabular}{|c||c||c|c|c|}
\hline Funct. & Benchmark & Best & Mean & Worst \\
\hline \hline g1 & -15.000 & -15.000 & -15.000 & -88.844 \\
\hline g2 & -0.803 & -0.803 & -0.796 & -0.064 \\
\hline g3 & -1.000 & -1.000 & -1.000 & -1.005 \\
\hline g4 & -30665.538 & -30665.538 & -30646.178 & -25186.227 \\
\hline g5 & 5126.496 & 5126.498 & 5240.496 & 4153.354 \\
\hline g6 & -6961.813 & -6961.825 & -6859.075 & -6482.554 \\
\hline g7 & 24.306 & 24.309 & 24.912 & 47.401 \\
\hline g8 & -0.095 & -0.095 & -0.095 & -72.264 \\
\hline g9 & 680.630 & 680.630 & 681.373 & 8825.132 \\
\hline g10 & 7049.248 & 7049.285 & 7850.401 & 1810.354 \\
\hline g11 & 0.749 & 0.749 & 0.749 & 1.361 \\
\hline g12 & -1.000 & -1.000 & -1.000 & -0.718 \\
\hline g13 & 0.053 & 0.054 & 0.450 & 1.082 \\
\hline g14 & -47.764 & -47.635 & -45.665 & -517.919 \\
\hline g15 & 961.715 & 961.715 & 962.516 & 768.737 \\
\hline g16 & -1.905 & -1.905 & -1.795 & 0.179 \\
\hline g17 & 8853.539 & 8853.539 & 8894.708 & 9003.614 \\
\hline g18 & -0.866 & -0.866 & -0.787 & 5.438 \\
\hline g19 & 32.655 & 34.018 & 64.505 & 55.835 \\
\hline g20 & 0.204 & 0.203 & 0.416 & 17.048 \\
\hline
\end{tabular}

TABLE III

Best Values obtained with AESSR Performing 500,000 OBJECTIVE FUNCTION EVALUATIONS.

\begin{tabular}{|c||c||c|c|c|}
\hline Funct. & Benchmark & Best & Mean & Worst \\
\hline \hline g1 & -15.000 & -15.000 & -15.000 & -15.000 \\
\hline g2 & -0.803 & -0.739 & -0.697 & -0.657 \\
\hline g3 & -1.000 & -1.000 & -1.000 & -1.000 \\
\hline g4 & -30665.538 & -30665.539 & -30665.539 & -30665.539 \\
\hline g5 & 5126.496 & 5126.497 & 5126.497 & 5126.497 \\
\hline g6 & -6961.813 & -6961.814 & -6961.814 & -6961.814 \\
\hline g7 & 24.306 & 24.306 & 24.307 & 24.312 \\
\hline g8 & -0.095 & -0.096 & -0.096 & -0.096 \\
\hline g9 & 680.630 & 680.630 & 680.630 & 680.630 \\
\hline g10 & 7049.248 & 7049.408 & 7061.087 & 7126.958 \\
\hline g11 & 0.749 & 0.750 & 0.750 & 0.750 \\
\hline g12 & -1.000 & -1.000 & -1.000 & -1.000 \\
\hline g13 & 0.053 & 0.054 & 0.116 & 0.439 \\
\hline g14 & -47.764 & -47.765 & -47.762 & -47.753 \\
\hline g15 & 961.715 & 961.715 & 961.715 & 961.715 \\
\hline g16 & -1.905 & -1.905 & -1.905 & -1.905 \\
\hline g17 & 8853.539 & 8853.540 & 8853.717 & 8857.816 \\
\hline g18 & -0.866 & -0.866 & -0.851 & 0.675 \\
\hline g19 & 32.655 & 32.665 & 32.829 & 33.242 \\
\hline g20 & 0.204 & - & - & - \\
\hline
\end{tabular}

solution (which is also infeasible) reported as the best known for this test function in [8].

Table II shows the Best, Mean and Worst values obtained by CPSO-shake for the 20 constrained test functions adopted in our empirical study.

Table III shows the Best, Mean and Worst values obtained by AESSR for the 20 constrained test functions adopted for our empirical study.

We also compared results with respect to the SR algorithm, using the same number of evaluations of the objective function $(350,000)$. We downloaded the source code of the SR algorithm from Runarsson's web page [7] and ran it ourselves
TABLE IV

COMPARISON OF THE best VALUES OBTAINED B Y OUR CPSO-SHAKE AND STOCHASTIC RANKING (SR).

\begin{tabular}{|c||c||c|c|}
\hline Funct. & Benchmark & CPSO-shake & SR \\
\hline \hline $\mathrm{g} 1$ & -15.000 & -15.000 & -15.000 \\
\hline $\mathrm{g} 2$ & -0.803 & -0.803 & -0.803 \\
\hline $\mathrm{g} 3$ & -1.000 & -1.000 & -1.000 \\
\hline $\mathrm{g} 4$ & -30665.539 & -30665.538 & -30665.539 \\
\hline $\mathrm{g} 5$ & 5126.496 & 5126.498 & 5126.497 \\
\hline $\mathrm{g} 6$ & -6961.813 & -6961.825 & -6961.814 \\
\hline $\mathrm{g} 7$ & 24.306 & 24.309 & 24.310 \\
\hline $\mathrm{g} 8$ & -0.095 & -0.095 & -0.095 \\
\hline $\mathrm{g} 9$ & 680.630 & 680.630 & 680.630 \\
\hline $\mathrm{g} 10$ & 7049.248 & 7049.285 & 7050.194 \\
\hline $\mathrm{g} 11$ & 0.749 & 0.749 & 0.749 \\
\hline $\mathrm{g} 12$ & -1.000 & -1.000 & -1.000 \\
\hline $\mathrm{g} 13$ & 0.053 & 0.054 & 0.053 \\
\hline $\mathrm{g} 14$ & -47.764 & -47.635 & -41.551 \\
\hline $\mathrm{g} 15$ & 961.715 & 961.715 & 961.715 \\
\hline $\mathrm{g} 16$ & -1.905 & -1.905 & -1.905 \\
\hline $\mathrm{g} 17$ & 8853.539 & 8853.539 & 8811.692 \\
\hline $\mathrm{g} 18$ & -0.866 & -0.866 & -0.866 \\
\hline $\mathrm{g} 19$ & 32.655 & 34.018 & 33.147 \\
\hline $\mathrm{g} 20$ & 0.204 & 0.203 & 0.057 \\
\hline
\end{tabular}

in order to perform a direct comparison of results. We had to implement the 7 last functions (from g14 to g20) and re-run the algorithm, since such functions were not available in the original source code. To implement those 7 functions we used the code available at [8]. The results obtained are compared with respect to our CPSO-shake and shown in Table IV. The best, mean and worst values obtained by SR are shown in Table V.

Table IV shows the best values found by our CPSO-shake and SR. The results of both algorithms are comparable: in 11 test functions both approaches reached the optimum (or best known solution). In 5 test functions (g7, g10, g14, g17 y g20), our CPSO-shake overperforms the SR algorithm, while in other 4 test functions ( $\mathrm{g} 4, \mathrm{~g} 5, \mathrm{~g} 6, \mathrm{~g} 13$ ) SR outperforms our CPSO-shake. However, it is important to emphasize that the previous version of CSPO, reported in [2], was able to outperforms SR only in one test function (g10). This is a clear indication of the improvements achieved by the new version of our PSO-based approach presented in this paper.

Figures 4, 5 and 6 show how far are the best values found by the algorithms with respect to the values reported to be the optima or best known for each test function. In particular, Figure 4 shows the best performance of the new algorithm presented in this paper with respect to our previous version, reported in [2].

\section{Statistical Analysis}

Another way to compare the performance of our algorithms is with a statistical test. The comparison with AESSR is not possible because we do not have the best values reached at each run. So, we performed an analysis of variance between SR and CPSO-shake using the best values of the 25 independent runs performed with each one. We applied the Kruskal-Wallis [6] nonparametric one-way analysis because 
TABLE V

Best VAlues obtained With SR PERFoRMing 350,000 obJeCtive FUNCTION EVALUATIONS.

\begin{tabular}{|c||c||c|c|c|}
\hline Funct. & Benchmark & Best & Mean & Worst \\
\hline \hline $\mathrm{g} 1$ & -15.000 & -15.000 & -15.000 & -15.000 \\
\hline $\mathrm{g} 2$ & -0.803 & -0.803 & -0.784 & -0.734 \\
\hline $\mathrm{g} 3$ & -1.000 & -1.000 & -1.000 & -1.000 \\
\hline $\mathrm{g} 4$ & -30665.538 & -30665.539 & -30665.480 & -30664.216 \\
\hline $\mathrm{g} 5$ & 5126.496 & 5126.497 & 5130.752 & 5153.757 \\
\hline $\mathrm{g} 6$ & -6961.813 & -6961.814 & -6863.645 & -6267.787 \\
\hline $\mathrm{g} 7$ & 24.306 & 24.310 & 24.417 & 24.830 \\
\hline $\mathrm{g} 8$ & -0.095 & -0.095 & -0.095 & -0.095 \\
\hline $\mathrm{g} 9$ & 680.630 & 680.630 & 680.646 & 680.697 \\
\hline $\mathrm{g} 10$ & 7049.248 & 7050.194 & 7423.434 & 8867.844 \\
\hline $\mathrm{g} 11$ & 0.749 & 0.750 & 0.750 & 0.751 \\
\hline $\mathrm{g} 12$ & -1.000 & -1.000 & -1.000 & -1.000 \\
\hline $\mathrm{g} 13$ & 0.053 & 0.053 & 0.061 & 0.128 \\
\hline $\mathrm{g} 14$ & -47.764 & -41.551 & -41.551 & -40.125 \\
\hline $\mathrm{g} 15$ & 961.715 & 961.715 & 961.731 & 962.008 \\
\hline $\mathrm{g} 16$ & -1.905 & -1.905 & -1.703 & -1.587 \\
\hline $\mathrm{g} 17$ & 8853.539 & 8811.692 & 8805.990 & 8559.613 \\
\hline $\mathrm{g} 18$ & -0.866 & -0.866 & -0.786 & -0.457 \\
\hline $\mathrm{g} 19$ & 32.655 & 33.147 & 34.337 & 37.477 \\
\hline g20 & 0.204 & 0.057 & 0.052 & 0.062 \\
\hline
\end{tabular}

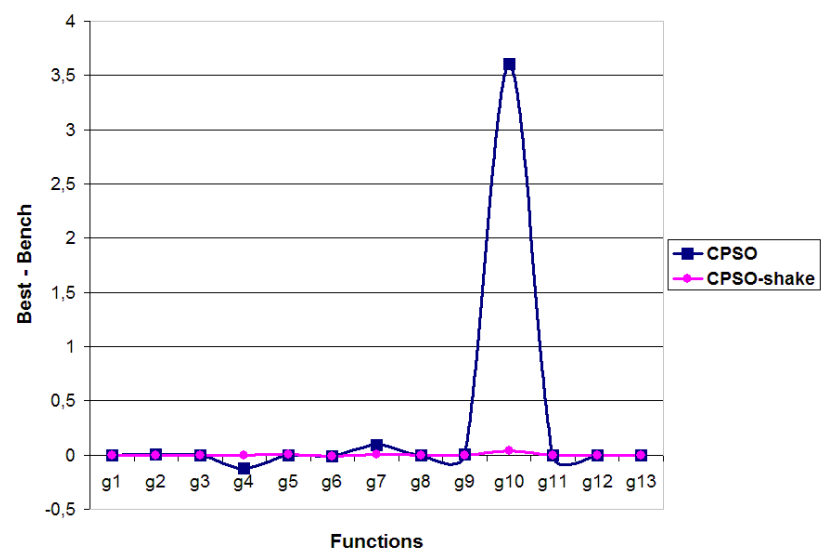

Fig. 4. Comparison between our original CPSO [2] and our CPSO-shake (this paper)

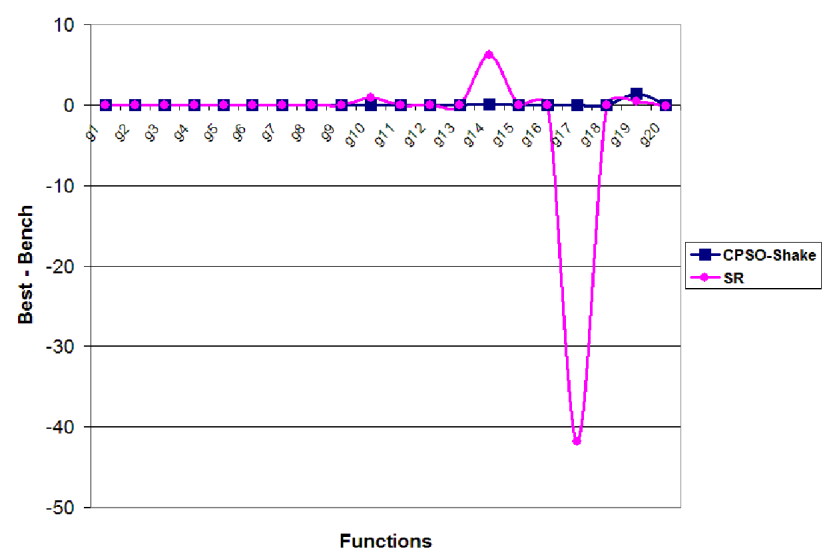

Fig. 5. Comparison between our CPSO-shake (this paper) and Stochastic Ranking (SR) [21]

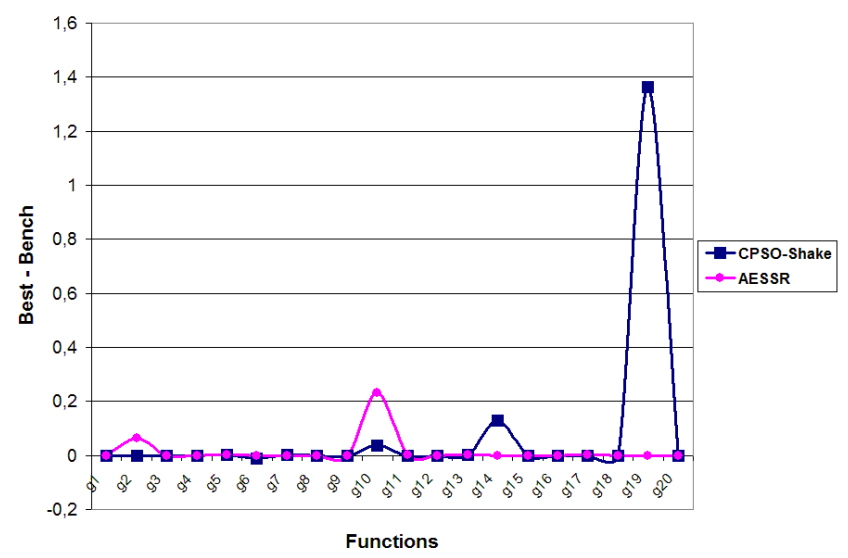

Fig. 6. Comparison between our CPSO-shake (this paper) and Approximate Evolution Strategy with Stochastic Ranking (AESSR) [20]

the values (the sample) do not have a normal distribution (determined with the Kolmogorov-Smirnov test).

The Kruskal-Wallis test returns the p-values for the null hypothesis for all samples. If the $p$-values are near zero, that suggests that at least one sample is significantly different (or statistically significant) than the other samples. Usually, if $p$-values are less than 0.05 , we declare that the results are significant.

Table VI shows the p-value for each function. The results indicate the values reached with CPSO-shake (for all functions except: g5, g12 and g16), are statistically significant from those of SR.

For those functions significantly different we apply the Tukey test to determine under which experimental conditions the differences are significant. The test returns: an estimate value (EV) into a range ([LI,LS]). If the range does not contain the zero-value, then the results are confirmed (significantly different). As we observe in Table VI, for every function statistically significant, the ranges do not have the zero-value.

\section{CONCLUSIONS}

In this paper, we have introduced an enhanced PSO algorithm called CPSO-shake, which is proposed to solve constrained numerical optimization problems. The results reached by CPSO-shake are very competitive with respect to Stochastic Ranking (which is one of the best constrainthandling techniques known to date) and to Approximation Evolution Strategy which uses Stochastic Ranking (recently proposed [20]) which is also a very competitive constrainthandling technique. Our preliminary results indicate that CPSO-shake is at least comparable to Stochastic Ranking and is also very satisfactory with respect to AESSR, since it obtained similar results despite performing $30 \%$ less objective function evaluations than AESSR. SR and AESSR presented a lower variability of the results than CPSO-shake, but this is due to the mechanisms implemented in our approach to maintain diversity. However, we argue that such mechanisms provide a tradeoff that we consider acceptable, since the best 
TABLE VI

KRUSKAL-WALLIS' P-VALUES AND TUKEY'S RESULTS FOR SR VS CPSO-SHAKE

\begin{tabular}{|c||c||c|c|c|}
\hline Funct. & $p$-value & LI & EV & LS \\
\hline \hline g1 & $9.00796 \mathrm{e}-011$ & 17.4420 & 25.0000 & 32.5580 \\
\hline g2 & 0.0011 & -20.9348 & -13.0800 & -5.2252 \\
\hline g3 & $1.28334 \mathrm{e}-010$ & 17.3787 & 25.0000 & 32.6213 \\
\hline g4 & $9.08263 \mathrm{e}-011$ & -32.5598 & -25.0000 & -17.4402 \\
\hline g5 & 0.6735 & - & - & - \\
\hline g6 & $5.35295 \mathrm{e}-005$ & -22.2776 & -15.0000 & -7.7224 \\
\hline g7 & $8.32678 \mathrm{e}-007$ & -26.5573 & -19.0000 & -11.4427 \\
\hline g8 & $2.55962 \mathrm{e}-012$ & -31.9998 & -25.0000 & -18.0002 \\
\hline g9 & $5.98833 \mathrm{e}-009$ & -30.8563 & -23.0800 & -15.3037 \\
\hline g10 & $6.75438 \mathrm{e}-009$ & -30.7763 & -23.0000 & -15.2237 \\
\hline g11 & 0.0003 & 6.5685 & 14.2917 & 22.0149 \\
\hline g12 & 1 & - & - & - \\
\hline g13 & $3.35698 \mathrm{e}-010$ & -32.6957 & -24.9200 & -17.1443 \\
\hline g14 & $6.75279 \mathrm{e}-010$ & 17.0609 & 25.0000 & 32.9391 \\
\hline g15 & $1.53751 \mathrm{e}-009$ & -32.7940 & -24.7600 & -16.7260 \\
\hline g16 & 0.2651 & - & - & - \\
\hline g17 & $3.60989 \mathrm{e}-010$ & -32.8147 & -25.0000 & -17.1853 \\
\hline g18 & $6.73625 \mathrm{e}-009$ & -30.7757 & -23.0000 & -15.2243 \\
\hline g19 & $3.3067 \mathrm{e}-010$ & -32.4829 & -24.7600 & -17.0371 \\
\hline g20 & $1.28627 \mathrm{e}-010$ & -32.6218 & -25.0000 & -17.3782 \\
\hline
\end{tabular}

values found by our approach remained very competitive despite the larger variability of results obtained (we sacrificed some online performance for the sake of improving offline performance).

Our proposed approach is one of the most competitive constraint-handling techniques currently available for the PSO algorithm (both SR and AESSR use an evolution strategy [22] as their search engine).

As part of our future work, we aim to improve the robustness of our CPSO-shake algorithm, by introducing alternative mechanisms to maintain diversity. We believe that this goal is achievable, but a much more careful study of the behavior of our PSO-based approach in constrained search spaces is required for that sake.

\section{ACKNOWLEDGMENT}

The third author gratefully acknowledges support from CONACyT project no. 45683-Y.

\section{REFERENCES}

[1] L. Cagnina, S. Esquivel, and R. Gallard. Particle swarm optimization for sequencing problems: a case study. In Congress on Evolutionary Computation, pages 536-541, Portland, Oregon, USA, 2004. http://www.lidic.unsl.edu.ar/publicaciones/info_publicacion.php?id_publicacion $=200$.

[2] L. C. Cagnina, Susana C. Esquivel, and C. A. Coello Coello. A particle swarm optimizer for constrained numerical optimization. In 9th International Conference - Parallel problem Solving from Nature - PPSN IX, pages 910-919, Reykjavik, Island, 2006.

[3] W. Cedeno and D. Agrafiotis. Particle swarms for drug design. In Proceeding of the IEEE Congress on Evolutionary Computation 2005, pages 479-486, Edinburgh, Scotland, 2005.

[4] E. Correa, A. Freitas, and C. Johnson. A new discrete particle swarm algorithm applied to attribute selection in a bioinformatics data set. In Proceeding of the GECCO 2006, pages 35-42, Seattle, Washington,USA, 2006.
[5] R. Eberhart and Y. Shi. A modified particle swarm optimizer. In International Conference on Evolutionary Computation, IEEE Service Center, Anchorage, AK, Piscataway, NJ, 1998.

[6] M. Hollander and D. A. Wolfe. Nonparametric Statistical Methods. Wiley, 1973.

[7] http://cerium.raunvis.hi.is/ tpr/software/sres/index.html.

[8] http://www.ntu.edu.sg/home/EPNSugan/index_files/CEC06/CEC06.html.

[9] X. Hu, R. Eberhart, and Y. Shi. Swarm intelligence for permutation optimization: a case study on n-queens problem. In Proceeding of the IEEE Swarm Intelligence Symposium, pages 243-246, Indianapolis, Indiana, USA, 2003.

[10] J. Kennedy. Small world and mega-minds: effects of neighborhood topologies on particle swarm performance. In 1999 Congress on Evolutionary Computation, pages 1931-1938, Piscataway, NJ, 1999. IEEE Service Center.

[11] J. Kennedy. Bores bones particle swarm. In IEEE Swarm Intelligence Symposium, pages 80-87, 2003.

[12] J. Kennedy and R. Eberhart. The particle swarm: social adaptation in information-processing systems. In D. Corne, M. Dorigo, and F. Glover, editors, New Ideas in Organization, 1999.

[13] J. Kennedy and R. Eberhart. Swarm Intelligence. Morgan Kaufmann Publishers, California, USA, 2001.

[14] J. Kennedy and W. Spears. Matching algorithm to problems: an experimental test of the particle swarm and some genetic algorithms on the multimodal problem generator. In IEEE Conference on Evolutionary Computation, Anchorage, Alaska, 1998.

[15] R. Krohling, H. Knidel, and Y. Shi. Solving numerical equations of hydraulic problems using particle swarm optimization. In Congress on Computational Intelligence, pages 1968-1961, Honolulu, Haway, 2002.

[16] J. J. Liang and P. N. Suganthan. Dynamic multi-swarm particle swarm optimizer with a novel constraint-handling mechanism. In IEEE Congress on Evolutionary Computation, pages 316-323, Vancouver, Canada, 2006. IEEE Press.

[17] Efrén Mezura-Montes and Carlos A. Coello Coello. A Simple Multimembered Evolution Strategy to Solve Constrained Optimization Problems. IEEE Transactions on Evolutionary Computation, 9(1):117, February 2005.

[18] Angel E. Muñoz et al. PESO+ for Constrained Optimization. In IEEE Congress on Evolutionary Computation, pages 936-942, Vancouver, Canada, 2006.

[19] G. Toscano Pulido and C. A. Coello Coello. A constraint-handling mechanism for particle swarm optimization. In IEEE Congress on Evolutionary Computation, pages 1396-1403, Portland, Oregon, USA, 2004.

[20] T. P. Runarsson. Approximate evolution strategy using stochastic ranking. In 2006 IEEE World Congress on Computation Intelligence, volume 3, pages 2760-2767, British Columbia, Canada, 2006.

[21] T. P. Runarsson and X. Yao. Stochastic ranking for constrained evolutionary optimization. In IEEE Transactions on Evolutionary Computation, volume 3, pages 284-294, 2000.

[22] Hans-Paul Schwefel. Evolution and Optimum Seeking. John Wiley \& Sons, New York, 1995.

[23] W. Zang, X. Xie, and D. Bi. Handling boundary constraints for numerical optimization by particle swarm flying in periodic search spaces. In IEEE Congress on Evolutionary Computation, pages 2307 2311, Portland, Oregon, USA, 2004. 